\title{
STUDENTS' PERCEPTION THAT PLAGIARISM IS EXAGGERATED BY EDUCATORS
}

\author{
V. Estivill-Castro, Geraldine Torrisi-Steele \\ Griffith University (AUSTRALIA)
}

\begin{abstract}
There is a large literature on academic dishonesty and plagiarism. Nevertheless, academic dishonesty is especially problematic in computer programming and information technology courses. Some suggest that the reasons why computer science and information technology seems to be more prone to academic misconduct are that assessment items often offer ideal solutions, and materials can be distributed and duplicated with ease. In the literature, there seems to be no study exploring whether the students believe that the issue of plagiarism is something exaggerated by the educators. In this research, we propose to explore to what extent students believe that once in their professional roles, the emphasis on plagiarism would dissipate.
\end{abstract}

Keywords: Plagiarism, Learning Outcomes, Ethics, teachers' role, industry practice.

\section{INTRODUCTION}

The market size of university and other higher education in Australia is now 34 billion Australian dollars. Domestic participation reached record levels when in 2016, 41\% of Australian 19-year-olds were enrolled in higher education institutions. But also, in 2016 overseas enrolments reached $20 \%$ of university enrolments. By 2018 , this increased to $25 \%$, and Australia is now yearly delivering programs to 400,000 international higher education students. This growth and the financial reliance by Australian universities on overseas fee-paying students has attracted media attention [18]. Naturally, because of the potential conflict of interest, the issue of maintaining standards and quality of education, while keeping in check academic misconduct and plagiarism have also attracted recent media attention $[5,3$, 6]. The concern for the industrialisation of cheating has also attracted attention from scholars who have been able to reveal that contract low-cost essays and assignments providers are indeed consolidating themselves [11].

There is a large literature on academic dishonesty and plagiarism [16]. It has been identified that academic dishonesty is especially problematic in computer programming and information technology courses $[1,10]$. Some attribute the apparently higher academic misconduct and plagiarism within computer science and information technology to the design of assessment items that often offer ideal solutions, and that materials can be distributed and duplicated with ease [8, 19, 4]. In the literature, some of the justifications or conditions that push students to academic misconduct are

1 the pace of the course and finding themselves without the assumed knowledge (even if all students in the course begin with equivalent skills) [15],

2 students may feel entitled to pass [4], such sense of entitlement may be derived because of the cost of the course or the amount of effort invested [4],

3 some students display counter-productive attitudes and poor practices (apathy, laziness, poor time management) [4],

4 students' lack of motivation or students' belief that the course or material is irrelevant [15].

Studies specific to STEM have focused on graduate students [12]. However, there seems to be no study exploring whether the students believe that the issue of plagiarism is something exaggerated by the educators. In this research, we propose to explore to what extent students believe that once in their professional roles, the emphasis on plagiarism would dissipate. Cheating and plagiarism is recognised as a global problem [20] and a major concern within Universities [20]; however, can this be attributed to the students belief that the issue is only relevant while studying? In other fields of study, such as business, the issue of plagiarism is consider relevant to professional practice [14]. However, others suggest that regularly fighting plagiarism is an unavoidable component of a professor occupation [2] as well as editors of learned journals. 


\section{METHODOLOGY}

This research aims at evaluating attitudes of IT and Computer Science students concerning plagiarism, to understand what is regarded as acceptable in a job or while studying, and whether there could be some actions that would be acceptable to students, including more information, or more policing.

We formulated a questionnaire applied to two types of respondents, alumni and current students of programs offered by the School of Information and Communication Technology at Griffith University. The research was conducted under Griffith University Human Research Ethics Committee project GU Ref No: 2019/943. We use SurveyMonkey Inc. to manage our surveys. Participants would be recruited by an invitation on Linkedln (alumni) and by e-mail (current students). The survey is exactly the same; however, the collector link is different, allowing the research team to identify whether the respondent is alumni or a current student. Except for the answers to the survey questions, no other information that could identify the respondent is collected. Thus, participants are aware their responses are anonymous, and they could stop at any time. Participants were informed of the details of the aims of the research, the contact data of the chief investigators and the task. We also provided clear indication that participation is voluntary, that they can stop at any time, and that the answers are completely anonymous. Survey Monkey ensures responses are completely anonymous. See Survey Monkey Privacy Policy (https://www.surveymonkey.com/mp/legal/privacy-policy/). The participants could keep the information sheet for future reference and what constitutes an indication of their consent. That is, in SurveyMonkey, the On-line survey presents the information sheet (which can be printed) and participants indicated their consent by explicitly pressing to continue. Participants were advised completing the survey could take about 30-35 minutes, but respondents typically completed it in under 20 minutes.

We used SurveyMonkey's capacity to randomise the questions; therefore, participants answered questions in different random order. The purpose of using this feature is to randomly separate parallel questions that are parallels of each other, one asking about the situation at a university while the other asking about the same situation but in a job. Questions in the survey request participants to complete a statement using a 5-point Likert Scale or a slider scale. The sliders could be converted to 5-point Likert Scale, but they also offer a sense of distance between responses (which is controversial with Likert Scales).

The null hypothesis typically will be that the respondents perceive the issues of plagiarism as serious in their professional practice as at University. The appendix includes the questionnaire. The tabulated anonymised questionnaire responses can be made available upon request, provided any further use of this data cites this publication.

\section{RESULTS}

We collected 38 surveys from alumni and 21 surveys from students; however, several did not complete the survey. Thus, we had a total of 29 complete surveys from alumni and 17 complete surveys from students. We focus first on the analysis of Question 12 which is the main focus of this paper. We use a diverging stacked bar charts for the data clustered in a 5-point Likert scale as suggested by Heiberger and Robbins [7].



Figure 1. Divergent plot for results in Question 12.

Figure 1 shows this plot. We can see that the students seems more inclined to disagree strongly with the statement of the question. That is, apparently students believe the issue of plagiarism is not exaggerated at universities; however, alumni seems to shift their mind and believe that what they 
experienced at university during their studies is somewhat distant from their real-world experience, where plagiarism matters matter less. The median for alumni's responses is Neutral although the mode is Strongly disagree; while for the students' responses moth the median and the mode is Strongly disagree. The original scale values result in a median for alumni's responses of 50 , but the median for students' responses is 16 . We hypothesise there is a significant difference between the mean response by alumni and the mean response by the current students. Thus, our null hypothesis is that the mean of the responses is the same. Our data is ordinal, and we cannot assume the samples come from normal distributions. Moreover, we have a total of $29+17=46$ response, that although larger than 30 could be considered a small sample (and the sample sizes of the two samples are not nearly the same). Nevertheless we use the t-test first because researchers have pointed out that the t-test is useful to avoid Type II error even in this case relative to a Wilcox Signed-Rank Test [13]. Also, others [9] suggest the t-test is still applicable in this case. But we use the $R$ [17] software and in particular its Welch Two Sample t-test as it is more reliable when the two samples have unequal variances and/or unequal sample sizes. We obtain that at the 95 percent confidence level (with $p$-value equal to 0.2322 ) that alumni have a view where their plagiarism in the work environment is less relevant that at universities. For further confirmation, we also used R [17] to perform a Mann-Whitney significance test, and found that also in this case we accept the alternative hypothesis that alumni find plagiarism less relevant in the work environment.

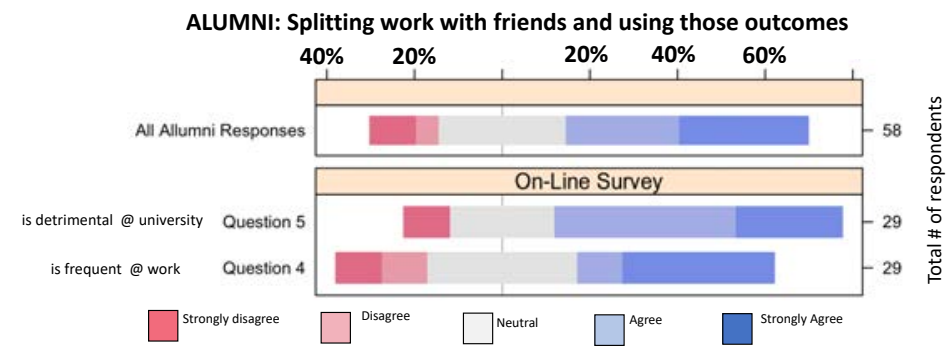

Figure 2. Divergent plot for alumni's responses for Question 4 and Question 5.

We compare the attitudes of alumni about an analogous activity such as splitting the work with others and using it, as evaluated by Question 4 and Question 5, where we contrast the work environment and the university environment. We observe, as illustrated by Figure 2 that splitting a task at work and using the contributions of friends is common (perhaps acceptable), but can be risky and result in some negative outcome if it happens at university.

\section{CONCLUSIONS}

The results above are reflective of some part of our thinking. Students and ICT professionals could potentially have different views on what is acceptable practices for claiming ownership of a solution to a problem. That is, there is a plethora of forums in the World-Wide-Web where questions are placed for community of software developers to respond and these are ranked according to their usefulness and accuracy. Organisations that release programming environments and software development tools also promote this practice to reduce the effort to write extensive tutorials or manuals. Therefore, there is a growing culture that the likelihood of finding the answer to a problem is already out-there and is probably more efficient to search for it that to work out the solution. Whether this culture on relying on resources from the World-Wide-Web is influencing or increasing the likelihood of plagiarism by ICT students remains open.

The earlier analysis is preliminary. We shall analyse the results of other questions and also attempt to see the correspondence with the length of time working outside university in the IT industry. We have received anecdotal evidence that plagiarism is on the rise. We already cited the recent reports in the media. However, at least three alumni contacted the researchers voluntarily and independently with remarks indicated they were unsatisfied with situations while students where they observed lenient attitudes to academic misconduct and a deterioration of the efforts to combat it. These concerns from alumni are an incentive to continue our research. 


\section{REFERENCES}

[1] C. Aasheim, P. Rutner, L. Li, and S. R. Williams. Plagiarism and programming: A survey of student attitudes. Journal of Information Systems Education, 23(3):298-313, 2012.

[2] J. J. Ballor. Plagiarism in a digital age. Journal of Markets \& Morality, 17(2), 2014.

[3] D. Bastian. Tehan introduces 'clarified' uni cheating laws to parliament. https://www.campusreview.com.au/2019/12/tehan-introduces-clarified-uni-cheating-laws-toparliament/. Accessed: 2019-20-12.

[4] R. Fraser. Collaboration, collusion and plagiarism in computer science coursework. Informatics in Education, 13(2):179-195, 2014.

[5] J. Fung. Chinese students paid to rort Australian universities as government tackles cheating. https://www.abc.net.au/news/2019-12-01/chinese-students-paid-to-ghost-write-for-australia-unistudents/11725330. Accessed: 2019-20-12.

[6] J. Goodman. Who's cheating at uni, and what are we going to do about it? https://www.campusreview.com.au/2019/11/whos-cheating-at-uni-and-what-are-we-going-to-doabout-it/. Accessed: 2019-20-12.

[7] Richard Heiberger and Naomi Robbins. Design of diverging stacked bar charts for Likert scales and other applications. Journal of Statistical Software, Articles, 57(5):1-32, 2014.

[8] M Hosny and S. Fatima. Attitude of students towards cheating and plagiarism: University case study. Journal of Applied Sciences, 14:748-757, 2014.

[9] A. Isaac and W. B Micheal. Handbook in Research and Evaluation - For Education and Behavioural Sciences. Educational and Industrial Testing Services, San Diego, CA., third edition, 1995.

[10] H. Jian, F. E. Sandnes, Y. Huang, L. Cai, and K. M. Y. Law. On students' strategy-preferences for managing difficult course work. IEEE Transactions on Education, 51(2):157-165, May 2008.

[11] T. Lancaster. Profiling the international academic ghost writers who are providing low-cost essays and assignments for the contract cheating industry. Journal of Information, Communication and Ethics in Society, 17(1):72-86, 2019.

[12] M. Leonard, D. Schwieder, A. Buhler, D. B. Bennett, and M. Royster. Perceptions of plagiarism by STEM graduate students: A case study. Science and Engineering Ethics, 21(6):1587-1608, 2015.

[13] G. E. Meek, C. Ozgur, and K. Dunning. Comparison of the t vs. Wilcoxon signed-rank test for Likert scale data and small samples. Journal of Modern Applied Statistical Methods, 6(1):91-106, May 2007.

[14] D. Nitterhouse. Plagiarism - not just an "academic" problem. Teaching Business Ethics, 7(3):215227, Aug 2003.

[15] D. J. Palazzo, Y.-J. Lee, R. Warnakulasooriya, and D. E. Pritchard. Patterns, correlates, and reduction of homework copying. Phys. Rev. ST Phys. Educ. Res., 6:010104, Mar 2010.

[16] C. Park. In other (people's) words: Plagiarism by university students-literature and lessons. Assessment \& Evaluation in Higher Education, 28(5):471-488, 2003.

[17] R Core Team. R: A Language and Environment for Statistical Computing. R Foundation for Statistical Computing, Vienna, Austria, 2013.

[18] N. Robinson. Australian universities risk catastrophe due to over-reliance on chinese students, expert warns. https://www.abc.net.au/news/2019-08-21/australian-universities-too-dependent-onchinese-students-report/11427272. Accessed: 2019-20-12.

[19] J. Sheard and M. Dick. Computing student practices of cheating and plagiarism: A decade of change. In Proceedings of the 16th Annual Joint Conference on Innovation and Technology in Computer Science Education, ITiCSE '11, pages 233-237, New York, NY, USA, 2011. ACM.

[20] J. Sheard, M. Dick, S. Markham, I. Macdonald, and M. Walsh. Cheating and plagiarism: Perceptions and practices of first year it students. In D. Finkel, editor, Proceedings: The 7th Annual Conference on Innovation and Technology in Computer Science Education, ITiCSE '02, pages 183 - 187, New York, NY, USA, 2002. Association for Computing Machinery (ACM). 


\section{APPENDIX: QUESTIONNAIRE}

1. To what extent do you believe you understand what the expectations for academic conduct are at the University

2. To what extent do you agree with the statement "Searching for a solution on the World Wide Web is something common in a job"

3. To what extent do you agree with the statement "Searching for a solution on the World Wide Web could get you in trouble in a University"

4. To what extent do you agree with the statement "Trading solutions and pieces of work with friends, effectively dividing the workload is something common in a job"

5. To what extent do you agree with the statement "Trading solutions and pieces of work with friends, effectively dividing the workload could get you in trouble in a University"

6. To what extent do you agree with the statement "Plagiarism is a severe matter."

7. To what extent do you believe you have been given comprehensive advice concerning plagiarism.

8. To what extent do you agree with the statement "Plagiarism is a complex issue."

9. To what extent do you agree with the statement "Posting to an Internet newsgroup for assistance is something common in a job."

10. To what extent do you agree with the statement "Posting to an Internet newsgroup for assistance could get you in trouble in a University."

11. To what extent do you agree with the statement "I feel confident to approach my tutor/convener to discuss plagiarism."

12. To what extent do you agree with the statement "Plagiarism is exaggerated at universities, in real jobs, nobody cares what the source was, what matters is to solve the task at hand."

13. To what extent do you agree with the statement "plagiarism is stealing intellectual material."

14. To what extent do you agree with the statement "to reference is to provide an acknowledgement, within your assignment, that the words used, or the ideas written about, in your work are not your own and belong to someone else."

15. To what extent do you agree with the statement "Collaborating with another person on a task meant to be completed individually is something common in a job."

16. To what extent do you agree with the statement "Collaborating with another person on a task meant to be completed individually could get you in trouble in a University."

17. To what extent do you agree with the statement "What is considered to constitute plagiarism is the opinion of each Subject Convenor and is usually different and not well-identified in the Subject Out-line or any other document you receive at the beginning of each subject."

18. To what extent do you agree with the statement "For an individual assignment, using other student's work/ideas/files/ without reference/acknowledgement is plagiarism."

19. To what extent do you agree with the statement "Resubmitting a piece of work that you produced and used before is something common in a job."

20. To what extent do you agree with the statement "Resubmitting a piece of work that you produced and used before could get you in trouble in a University."

21. To what extent do you agree with the statement "Providing work to another student who hands it in as their own is not plagiarism, the guilty one is the other student."

22. To what extent do you agree with the statement "Plagiarism is justifiable if you are running out of time or if you experience any circumstances beyond your control such as illness or misadventure."

23. To what extent do you agree with the statement "There is little difference between (1) telling someone how to approach a problem or (2) writing, for example, a nearly full solution, or an essay, that would enable someone to submit work they did not completely understand." 
24. To what extent do you agree with the statement "An international student can't manage the workload and assignments on top of the language and cultural barriers; thus, the university should be lenient in these cases concerning plagiarism."

25. To what extent do you agree with the statement "Copying the majority of a report from a friend's piece of work, but doing a fair bit of work oneself is something common in a job."

26. To what extent do you agree with the statement "Copying the majority of a report from a friend's piece of work, but doing a fair bit of work oneself could get you in trouble in a University."

27. To what extent do you agree with the statement "Plagiarism can be avoided by starting early, working hard, resolve misunderstandings by consulting tutors/teachers and by referencing other's work."

28. To what extent do you agree with the statement "Copying material from a textbook is something common in a job."

29. To what extent do you agree with the statement "Copying material from a textbook could get you in trouble in a University."

30. To what extent do you agree with the statement "In the School of ICT, there is insufficient education on plagiarism and insufficient policing."

31. To what extent do you agree with the statement "In the School of ICT, it is more common for students to plagiarise than to ask for more time or Special Consideration if necessary."

32. To what extent do you agree with the statement "Hiring a person to do your work and produce an outcome (you will later claim the outcome as your own) is something common in a job (since you paid for such outcome)."

33. To what extent do you agree with the statement "Hiring a person to do your work and obtain some outcome (you will later claim the outcome as your own since you paid for it) could get you in trouble in a University."

34. To what extent do you agree with the statement "In the School of ICT, students will rather plagiarise because there is a high chance it will not be detected, and when detected the penalty is small, for example, one may request to repeat the task and resubmission is usually granted."

35. To what extent do you agree with the statement "In the School of ICT, students are expected to work productively on the items of assessment of each course and progress in the course is evaluated by measuring the merit by the individual student work."

36. To what extent do you agree with the statement "Using a hidden sheet of paper with important facts during (a) a University exam or (b) a technical exam in a recruitment process for a job are equally bad."

37. I would support stronger policing and enforcement of the universities policy on plagiarism.

38. I would support further clarification on what constitutes plagiarism.

39. To what extent do you agree with the statement "Obtaining a medical certificate from a doctor to get an extension (when you are not actually sick) is something common in a job and not punishable if discovered."

40. To what extent do you agree with the statement "Obtaining a medical certificate from a doctor to get an extension (when you are not actually sick) could get you in trouble in a University if discovered."

41. To what extent do you agree with the statement "As a student, I have a responsibility to eradicate a culture that favours plagiarism."

42. To what extent do you agree with the statement "In the School of ICT, it is hard to make sure I have the required knowledge to attempt a course, and by the time this becomes apparent I am forced to bend corners concerning plagiarism."

43. I believe the School of ICT has a culture where plagiarism is tolerated.

44. I believe the School of ICT has a culture where the amount of plagiarism that is happening does not affect the prestige of the degrees. 
45. To what extent do you believe you understand what the expectations for intellectual property and work ethic are at jobs in the It Industry

46. Please indicate (i) currently studying first year (ii) currently studying second year (iii) currently studying third year (iv) currently studying honours (v) currently studying a masters (vi) currently studying a PhD (vii) Other (please specify)

47. Please indicate your work experience (i) I do not have experience with a job in the IT industry (ii) some experience with an IT job, but less than 9 months (iii) approximately one year ( 9 months to 18 months) (iv) a few years (1.5 years to less than 3 years) (v) several years ( 3 years to less than 6 years) (vi) more than 6 years 